\title{
A functional granulocyte colony-stimulating factor receptor is required for normal chemoattractant-induced neutrophil activation
}

\author{
Tomoko Betsuyaku, ${ }^{1}$ Fulu Liu, ${ }^{2}$ Robert M. Senior, ${ }^{1}$ Jeffery S. Haug, ${ }^{2}$ Eric J. Brown, ${ }^{3}$ \\ Samuel L. Jones, ${ }^{4}$ Kouji Matsushima, ${ }^{5}$ and Daniel C. Link ${ }^{2}$ \\ ${ }^{1}$ Division of Pulmonary and Critical Care Medicine, \\ ${ }^{2}$ Division of Bone Marrow Transplantation and Stem Cell Biology, and \\ ${ }^{3}$ Division of Infectious Diseases, Department of Internal Medicine, Washington University School of Medicine, St. Louis, Missouri \\ 63110-1093, USA \\ ${ }^{4}$ Department of Food Animal and Equine Medicine, College of Veterinary Medicine, North Carolina State University, Raleigh, North \\ Carolina, 27606, USA \\ ${ }^{5}$ Department of Molecular Preventive Medicine, Graduate School of Medicine, University of Tokyo, Tokyo, Japan 113-8654
}

Address correspondence to: Daniel C. Link, Washington University School of Medicine, Division of Bone Marrow Transplantation and Stem Cell Biology, Campus Box 8007, 660 South Euclid Avenue, St. Louis, Missouri 63110-1093, USA. Phone: (314) 362-8771; Fax: (314) 362-9333; E-mail: dlink@im.wustl.edu

This study was presented in preliminary form at the annual ALA/ATS International Conference in Chicago,Illinois, USA, April 26, 1998. Portions of this work have appeared in abstract form (1998. Am. J. Respir. Crit. Care Med. 157:28a).

Tomoko Betsuyaku and Fulu Liu contributed equally to this work.

Received for publication September 10, 1998, and accepted in revised form February 1, 1999.

Granulocyte colony-stimulating factor (G-CSF) is a hematopoietic growth factor that is widely used to treat neutropenia. In addition to stimulating polymorphonuclear neutrophil (PMN) production, G-CSF may have significant effects on PMN function. Because G-CSF receptor (G-CSFR)-deficient mice do not have the expected neutrophilia after administration of human interleukin-8 (IL-8), we examined the effect of the loss of G-CSFR on IL-8-stimulated PMN function. Compared with wild-type PMNs, PMNs isolated from G-CSFR-deficient mice demonstrated markedly decreased chemotaxis to IL-8. PMN emigration into the skin of G-CSFR-deficient mice in response to IL-8 was also impaired. Significant chemotaxis defects were also seen in response to $N$-formyl-methionyl-leucyl-phenylalanine, zymosan-activated serum, or macrophage inflammatory protein-2. The defective chemotactic response to IL- 8 does not appear to be due to impaired chemoattractant receptor function, as the number of IL-8 receptors and chemoattractant-induced calcium influx, actin polymerization, and release of gelatinase B were comparable to those of wild-type PMNs. Chemoattractant-induced adhesion of G-CSFR-deficient PMNs was significantly impaired, suggesting a defect in $\beta 2$-integrin activation. Collectively, these data demonstrate that selective defects in PMN activation are present in G-CSFR-deficient mice and indicate that G-CSF plays an important role in regulating PMN chemokine responsiveness.

J. Clin. Invest. 103:825-832 (1999).

\section{Introduction}

Polymorphonuclear neutrophils (PMNs) are essential cells in the host defense against a variety of infectious agents. Circulating PMNs require activation to migrate to inflammatory sites and efficiently kill pathogens. Data suggest that chemokines, in particular interleukin-8 (IL-8), play an important role in the activation and migration of PMNs (1). Consistent with this view, IL-8 is frequently detected at inflammatory sites characterized by PMN invasion (2). IL8 is a potent activator of PMNs in vitro, leading to enhanced chemotaxis, degranulation, adherence, and superoxide generation (2). In addition, IL-8 activates PMNs at inflammatory sites (3). Granulocyte colony-stimulating factor (GCSF) has been shown to upregulate IL-8 receptor expression on PMNs, suggesting a role for G-CSF in modulating IL-8-stimulated PMN responses (4).

G-CSF is the principal hematopoietic growth factor regulating the production of PMNs (5). It is widely used to treat neutropenia in a variety of clinical settings. Sub- stantial evidence suggests that, in addition to promoting PMN production, G-CSF may play a significant role in regulating mature $\mathrm{PMN}$ functions, including $\mathrm{CD} 11 \mathrm{~b}$ (6-8) and FcyR1 (9-11) expression, degranulation $(12,13)$, phagocytosis (14), adhesion (6-8), and chemokinesis (15, 16). In addition, pretreatment of PMNs with G-CSF renders the cells more responsive to activating agents. For example, G-CSF-treated ("primed") PMNs have an enhanced superoxide burst in response to the chemotactic peptide $N$-formyl-methionyl-leucyl-phenylalanine (fMLP) or complement fragments $(8,17,18)$. Because GCSF levels are often elevated in the serum and at inflammatory sites in septic patients $(19,20)$, it has been proposed that G-CSF may modulate PMN activation in vivo.

The actions of G-CSF are mediated through its interaction with the G-CSF receptor (G-CSFR). To define further the role of G-CSF in the regulation of PMN production, maturation, and function, we previously generated G-CSFR-deficient mice. Like G- 
CSF-deficient mice (21), G-CSFR-deficient mice have chronic neutropenia $(22,23)$. No accumulation of immature myeloid precursors in the bone marrow is present, suggesting that granulocytic differentiation of the residual myeloid precursors is normal $(22,23)$. Initial studies (22) suggested that the PMNs in GCSFR-deficient mice were phenotypically normal because their morphology; expression of CD11b, Gr1 , and myeloperoxidase (MPO); and migration into the peritoneum in response to thioglycollate were normal. However, a more recent study (24) showed that IL-8-induced mobilization of PMNs into the blood was absent in G-CSFR-deficient mice, suggesting a defect in IL-8-induced PMN migration. To investigate this possibility, we have examined the activation and function of G-CSFR-deficient PMNs. We show that significant but selective defects in PMN activation are present in G-CSFR-deficient mice, suggesting that the G-CSFR provides unique signals required for normal PMN function.

\section{Methods}

Materials. AMLP, MPO (EC 1.11.1.7), and zymosan were purchased from Sigma Chemical Co. (St. Louis, Missouri, USA). Thioglycollate broth was purchased from Difco Laboratories (Detroit, Michigan, USA). Recombinant human IL-8 was a gift from Monsanto/Searle Co. (St. Louis, Missouri, USA). Zymosan-activated serum (ZAS) was generated as described (25). Murine macrophage inflammatory protein-2 (MIP-2) was obtained from R\&D Systems Inc. (Minneapolis, Minnesota, USA). Anti-murine IL-8 receptor orthologue antibody (26) and monoclonal antibodies 5C6 (anti-murine CD11b; ref. 27) and mIAP301 (anti-murine IAP, CD47; ref. 28) have been described previously.

G-CSFR-deficient mice. G-CSFR-deficient mice (outbred $\mathrm{C} 57 \mathrm{BL} / 6 \times 129 \mathrm{SvJ}$ ) were generated in our laboratory as described previously (22). In some experiments, G-CSFR-deficient mice on a pure $129 \mathrm{SvJ}$ genetic background were used. All mice were housed in a specific pathogen-free environment. In this environment, G-CSFR-deficient mice are indistinguishable from wild-type; no increased incidence of clinical infections was observed. Six-to 10-week-old wild-type and G-CSFR-deficient mice were used for studies. All experiments were approved by the Washington University Animal Studies Committee.

Isolation of PMNs. PMNs were purified from bone marrow of mice using a discontinuous Percol gradient as described previously (29). Unless otherwise stated in the text, PMN purity (as assessed by examination of Wright-stained cytospin preparations) was $75 \%-85 \%$ for wild-type preparations and $40 \%-60 \%$ for G-CSFR-deficient preparations. Thioglycollate-elicited PMNs were harvested from the peritoneal space $4 \mathrm{~h}$ after an intraperitoneal injection of $1 \mathrm{ml}$ of thioglycollate. PMN purity was $75 \%-85 \%$ and $50 \%-60 \%$ for wild-type and G-CSFR-deficient preparations, respectively.

Chemotaxis assay. PMN chemotaxis was quantified using a modification of the Boyden chamber technique (30). A cell suspension containing $3.0 \times 10^{6} \mathrm{PMN} / \mathrm{ml}$ (the total cell number loaded per well was adjusted to give equal numbers of PMNs) in HBSS with $1 \mathrm{mM} \mathrm{CaCl}_{2}, 1 \mathrm{mM} \mathrm{MgCl}_{2}$ containing $0.1 \% \mathrm{BSA}$, was placed in the top wells of a 48-well microchemotaxis chamber (Neuro Probe Inc., Bethesda, Maryland, USA). A polyvinylpyrolidone (PVP)-free polycarbonate filter ( $3-\mu \mathrm{m}$ pore size; Poretics Products, Livermore, California, USA) separated the cells from lower wells containing the indicated chemoattractant. The chamber was incubated for $90 \mathrm{~min}$ at $37^{\circ} \mathrm{C}$ in a $5 \% \mathrm{CO}_{2}$ humidified atmosphere. After incubation, the filter was stained with LeukoStat (Fisher Scientific Co., Pittsburgh, Pennsylvania, USA), and the number of PMNs on the undersurface of the filter was counted in five random high-power fields $(\times 400)$ for each of triplicate filters.

IL-8 receptor orthologue expression. Bone marrow mononuclear cells were incubated with FITC-conjugated anti-Gr-1 (PharMingen, San Diego, California, USA) and the $\mathrm{F}\left(\mathrm{ab}^{\prime}\right)_{2}$ fragments of either rabbit anti-mouse IL-8 receptor orthologue

\section{$a$}

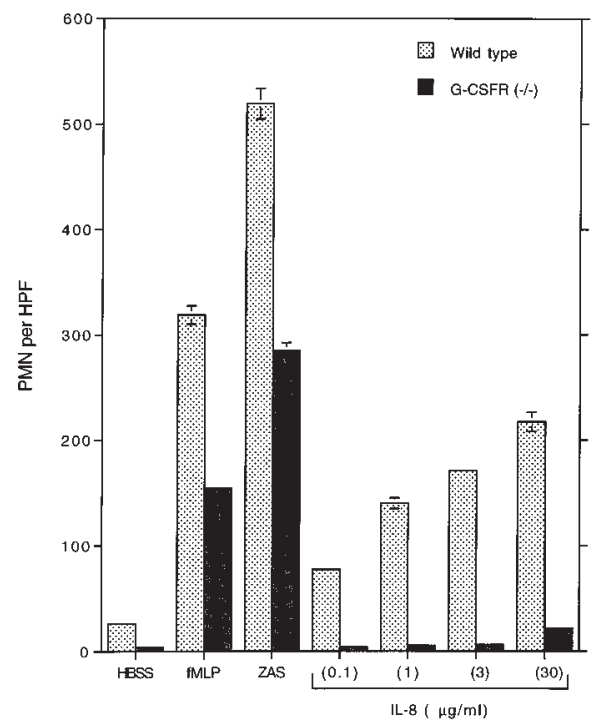

$b$

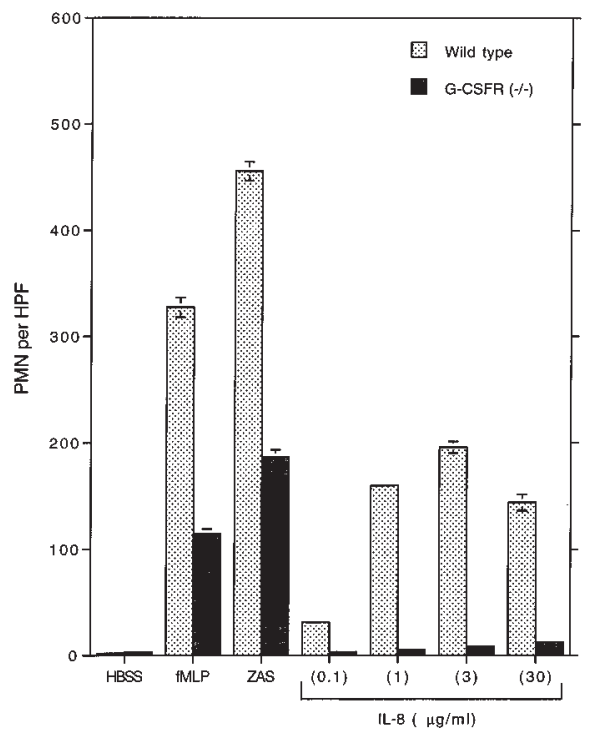

\section{Figure 1}

Granulocyte colony-stimulating factor receptor (G-CSFR)-deficient PMN chemotaxis. PMN chemotaxis was determined in modified Boyden chambers with bone marrow-purified PMNs (a) or thioglycollate-elicited peritoneal PMNs (b). Buffer alone (HBSS), $N$-formyl-methionyl-leucyl-phenylalanine ( $F M L P ; 0.1 \mathrm{mM}$ ), zymosan-activated serum (ZAS; $5 \% \mathrm{vol} / \mathrm{vol})$, or the indicated amount of human interleukin-8 (IL-8) was used as chemoattractant. The average number of PMNs per high-power field (PMN per HPF) \pm SE of triplicate samples is shown. Data are representative of three separate experiments. PMN, polymorphonuclear neutrophil. 
antibody or control rabbit antisera (26). Subsequently, cells were incubated with phycoerythrin $(\mathrm{PE})$-conjugated goat anti-rabbit antibody and analyzed using a FACScan flow cytometer (Becton Dickinson, Mansfield, Massachusetts, USA) and CellQuest version 1.2.2 software (Becton Dickinson).

Chemoattractant-induced calcium influx. Bone marrow neutrophils were loaded with the calcium-sensitive dye fluo-3 (9 $\mu \mathrm{M}$ acetoxymethyl ester fluo-3; Molecular Probes Inc., Eugene, Oregon, USA) for $30 \mathrm{~min}$ at room temperature in HBSS. Cells were stained with PE-conjugated anti-Gr-1 antibody, washed, and then resuspended in HEPES buffer $(137 \mathrm{mM} \mathrm{NaCl}, 5 \mathrm{mM}$ $\mathrm{KCl}, 1 \mathrm{mM} \mathrm{Na}_{2} \mathrm{HPO}_{4}, 5 \mathrm{mM}$ glucose, $1 \mathrm{mM} \mathrm{CaCl}_{2}, 0.5 \mathrm{mM}$ $\mathrm{MgCl}_{2}, 0.1 \% \mathrm{BSA}$, and $10 \mathrm{mM}$ HEPES; pH 7.4). Anti-fluorescein antibody was added per manufacturer's recommendations (Molecular Probes Inc.) to quench extracellular fluo-3 signals. The indicated agonist was added, and cellular fluo-3 fluorescence was measured continuously for $90 \mathrm{~s}$ using a Coulter ESP flow cytometer (Coulter Electronics Ltd., Hialeah, Florida, USA). Data represent the mean fluorescent intensity of 5-s intervals and are gated for Gr-1-positive cells.

Gelatinase B degranulation. Bone marrow PMNs in HBSS with $1 \mathrm{mM} \mathrm{CaCl}_{2}, 1 \mathrm{mM} \mathrm{MgCl}_{2}$, and $0.1 \% \mathrm{BSA}$ were incubated with the indicated chemoattractant at $37^{\circ} \mathrm{C}$ for $5 \mathrm{~min}$. Supernatants were collected after centrifugation at $500 \mathrm{~g}$ for $5 \mathrm{~min}$ and stored at $-70^{\circ} \mathrm{C}$. The gelatinase $\mathrm{B}$ present in the supernatant was measured by gelatin zymography as described previously (31).

CD11b expression. Bone marrow PMNs were incubated with PE-conjugated CD11b (Mac-1) or isotype control (PharMingen) for $5 \mathrm{~min}$ at $37^{\circ} \mathrm{C}$ and then stimulated with buffer, IL-8 (3 $\mu \mathrm{g} / \mathrm{ml})$, or fMLP $(0.1 \mathrm{mM})$ for $10 \mathrm{~min}$ at $37^{\circ} \mathrm{C}$. Cells were promptly transferred to $4^{\circ} \mathrm{C}$, incubated with FITC-conjugated anti-Gr-1 in the presence of sodium azide (0.2\%), and analyzed using a FACScan flow cytometer (Becton Dickinson). Results were gated for Gr-1-positive cells.

Superoxide generation. Superoxide anion $\left(\mathrm{O}^{2-}\right)$ generation was measured as described (32). Briefly, $1.5 \times 10^{5}$ bone marrow PMNs suspended in HBSS with $1 \mathrm{mM} \mathrm{CaCl} 2,1 \mathrm{mM} \mathrm{MgCl}_{2}$, and $50 \mu \mathrm{M}$ ferricytochrome $\mathrm{C}$ (Sigma Chemical Co.) were incubated in 96-well plates at $37^{\circ} \mathrm{C}$ for $3 \mathrm{~min}$. The $\mathrm{OD}_{550}$ was measured before and after incubation with agonist at $37^{\circ} \mathrm{C}$. As controls, duplicate wells were incubated in the presence of superoxide dismutase (180 U/ml; Sigma Chemical Co.). All analyses were performed using an Emax microplate reader (Molecular Devices, Sunnyvale, California, USA).

PMN adhesion. PMN adhesion to FBS-coated plates was measured as described previously (33). Bone marrow PMNs were loaded with calcein-AM (Molecular Probes Inc.) at 1-2 $\mu \mathrm{g} / \mathrm{ml}$ for $30 \mathrm{~min}$ at room temperature. After washing, PMNs were resuspended at $2 \times 10^{6} / \mathrm{ml}$ in HBSS with $0.1 \%$ BSA (the total cell number was adjusted to give equal numbers of PMNs). A total of $50 \mu \mathrm{l}$ of the PMN suspension was layered onto FBS-coated 96-well Immulon 2 plates (Dynatech, Chantilly, Virginia, USA), allowed to settle for $6 \mathrm{~min}$ at room temperature, and then incubated with the indicated agonist at $37^{\circ} \mathrm{C}$. At the indicated time, fluorescence was read at Ex 485/Em $538 \mathrm{~nm}$ using an fMax fluorescence plate reader (Molecular Devices). The wells were then washed twice with PBS to remove nonadherent PMNs, and the fluorescence was remeasured. The adhesion index is the fluorescence after washing divided by the fluorescence before washing $(\times 100)$ and represents the percentage of adherent cells.

Chemoattractant-induced F-actin polymerization. Bone marrow PMNs were incubated with PE-conjugated anti-Gr-1 antibody, washed, and then stimulated with IL-8 $(3 \mu \mathrm{g} / \mathrm{ml})$ at $37^{\circ} \mathrm{C}$ for the indicated times. Cells were simultaneously fixed and stained with a FITC-phalloidin solution $\left(4.0 \times 10^{-7} \mathrm{~mol} / \mathrm{l}\right.$ FITCphalloidin [Molecular Probes Inc.], $0.5 \mathrm{mg} / \mathrm{ml} 1-\alpha$-lysophosphatidylcholine [Sigma Chemical Co.], and 18\% formaldehyde [Sigma Chemical Co.]) as described previously (34). The mean fluorescence of Gr-1-positive cells was measured using a FACScan flow cytometer (Becton Dickinson).

PMN emigration into the skin. IL-8 (50 ng in $50 \mu \mathrm{l}$ of PBS with $0.1 \%$ endotoxin-free BSA) or PBS with BSA was injected intradermally into the abdomen of mice. In some experiments, mice were given both IL-8 and PBS injections at well-separated sites. Four hours after injection, the skin at the injection site $(\sim 0.25$ $\mathrm{cm}^{2}$ ) was excised and homogenized in buffer containing $0.1 \mathrm{M}$ Tris- $\mathrm{Cl}$ ( $\mathrm{pH}$ 7.6), $0.15 \mathrm{M} \mathrm{NaCl}$, and $0.5 \%$ hexadecyl trimethyl ammonium bromide. MPO activity was measured by the change in $\mathrm{OD}_{450}$ resulting from the decomposition of $\mathrm{H}_{2} \mathrm{O}_{2}$ in the presence of $o$-dianisidine (Sigma Chemical Co.) (35). The results were normalized for total protein content using the BioRad dye binding assay (Bio-Rad Laboratories Inc., Hercules, California, USA). A portion of the excised skin was fixed in $10 \%$ buffered formalin for $24 \mathrm{~h}$, embedded in paraffin, sectioned at $5 \mu \mathrm{m}$, and stained with hematoxylin and eosin $(H \& E)$.

\section{Results}

G-CSFR-deficient PMNs have impaired chemotaxis. The failure to mobilize PMNs into the peripheral circulation of GCSFR-deficient mice after IL-8 administration suggested a defect in IL-8-induced PMN migration. To test this hypothesis, we measured PMN chemotaxis in response to IL-8, the chemotactic peptide fMLP, ZAS (a source of C5a), or MIP-2. Morphologically mature PMNs isolated from the bone marrow of G-CSFR-deficient mice demonstrated significantly reduced chemotaxis in response to AMLP, ZAS, or MIP-2 and nearly absent chemotaxis in response to IL-8 (Fig. 1a, and data not shown). Similar results were obtained with PMNs isolated from the bone marrow of inbred 129SvJ G-CSFR-deficient mice (data not shown), demonstrating that the chemotactic defect was due to the loss of the G-CSFR and not mouse strain differences. Because G-CSFR-deficient mice have decreased numbers of mature PMNs in their bone marrow, it is possible that PMNs isolated from the bone marrow of these mice may not be fully mature. To address this issue, we measured the chemotactic response of thioglycollate-elicited peritoneal PMNs. Although quantitatively reduced relative to wildtype mice, thioglycollate injection into the peritoneum of G-CSFR-deficient mice results in the recruitment of a similar uniform population of mature-appearing PMNs (22). These PMNs also demonstrated a decreased chemotactic response to AMLP, ZAS, or MIP-2 and a nearly absent response to IL-8 (Fig. 1b, and data not shown). Collectively, these data show that the G-CSFR is required for normal neutrophil chemotaxis in vitro.

Mouse IL-8 receptor orthologue expression is normal in GCSFR-deficient PMNs. A report (4) suggested that G-CSF may regulate the expression of both human IL-8 receptors (CXCR1 and CXCR2). In mice, a single receptor for human IL-8 has been identified (36-38). To determine whether reduced IL-8 receptor orthologue expression could account for the impaired IL-8-induced PMN chemotaxis, we examined IL-8 receptor expression on GCSFR-deficient PMNs. Flow cytometric analysis of bone marrow PMNs using a specific anti-murine IL-8 receptor orthologue antibody (4) demonstrated a similar pattern of binding, indicating that IL-8 receptor orthologue expression on G-CSFR-deficient PMNs is normal (Fig. 2). Chemoattractant-induced calcium influx is normal in GCSFR-deficient PMNs. We next examined the ability of G- 

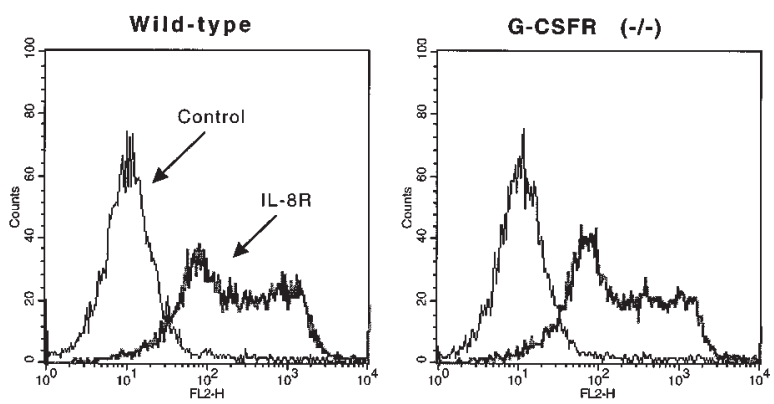

Figure 2

IL-8 receptor orthologue expression. Bone marrow-purified PMNs were incubated with anti-murine IL-8 receptor orthologue antibody or control antibody and analyzed by flow cytometry. Results are gated for Gr-1-positive (granulocytic) cells. Data are representative of three separate experiments.

CSFR-deficient PMNs to influx calcium after chemoattractant stimulation. Calcium influx is an early biochemical marker of chemoattractant signaling and requires a functional chemoattractant receptor. The chemoattractant-induced increase in cytoplasmic free $\mathrm{Ca}^{2+}$ in bone marrow PMNs was examined by flow cytometry. The kinetics and magnitude of calcium influx in GCSFR-deficient PMNs in response to IL-8, AMLP, or ZAS were comparable to those in wild-type PMNs (Fig. 3). Furthermore, G-CSFR-deficient PMNs demonstrated a similar IL-8 dose-response relationship as did wild-type PMNs (data not shown). These data provide evidence that the expression and function of chemoattractant receptors in G-CSFR-deficient neutrophils are normal.

Superoxide generation in response to phorbol myristate acetate is normal in G-CSFR-deficient PMNs. G-CSF treatment primes PMNs to generate superoxide in response to chemoattractant stimulation $(8,17,18)$. We therefore examined the effect of the loss of G-CSFR signals on the generation of superoxide by PMNs. In response to phorbol myristate acetate (PMA), superoxide generation in GCSFR-deficient bone marrow PMNs was comparable to that of wild-type PMNs (Fig. 4). Unfortunately, we were unable to detect superoxide generation consistently after
fMLP, ZAS, IL-8, or MIP-2 stimulation of either wildtype or G-CSFR-deficient bone marrow PMNs.

Chemoattractant-induced degranulation of secondary and tertiary granules is normal in G-CSFR-deficient PMNs. An important functional property of PMNs is their ability to release granule contents in response to agonists. We examined two separate measures of PMN degranulation: gelatinase B release and upregulation of cell surface CD11b (Mac-1) expression. Gelatinase B is expressed in PMNs and is stored in secondary and tertiary (gelatinase) granules (39). Treatment of wild-type PMNs with IL-8 or fMLP resulted in the substantial release of gelatinase $B$ into the supernatant as measured by gelatin zymography (Fig. 5 a). A similar pattern was observed with G-CSFR-deficient PMNs. CD11b is the major $\beta 2$ integrin expressed in PMNs and is stored in secondary and tertiary granules and secretory vesicles $(39,40)$. Stimulation of wild-type or G-CSFR-deficient PMNs with IL-8 or fMLP induced a similar upregulation of cell surface CD11b expression (Fig. 5b). Collectively, these data suggest that chemoattractant-induced release of secondary and tertiary granules is normal in G-CSFR-deficient PMNs.

Adhesion is defective in G-CSFR-deficient PMNs. The adhesion of PMNs to a surface is an early step in chemotaxis. Relative to wild-type PMNs, stimulation of GCSFR-deficient PMNs with IL-8, fMLP, or ZAS induced substantially less adhesion at early (three minutes after stimulation) time points (Fig. 6). Likewise, PMA-induced adhesion was also decreased in G-CSFR-deficient PMNs. As reported previously (33), incubation of cells with an anti- $\beta 2$ integrin antibody, but not an antibody directed against CD47, substantially reduced the adhesion of both wild-type and G-CSFR-deficient PMNs (data not shown), demonstrating an important role for $\beta 2$ integrins in PMN adhesion. Collectively, these data show that chemoattractant- and PMA-induced rapid adhesion of G-CSFR-deficient PMNs is impaired and suggest that $\beta_{2}$-integrin activation is defective.

Chemoattractant-induced F-actin polymerization is normal in GCSFR-deficient PMNs. The ability of agonists to induce actin polymerization is essential for cell adhesion and chemotaxis. IL-8-induced increases in F-actin content in bone

\section{Figure 3}

Chemoattractant-induced calcium influx. Bone marrow-purified PMNs were loaded with the calcium sensitive dye fluo-3 and stimulated (at time 0 ) with buffer alone (control) or the indicated stimulant. Changes in cytoplasmic free $\mathrm{Ca}^{2+}$ were measured continuously by flow cytometry. Data are representative of three separate experiments.
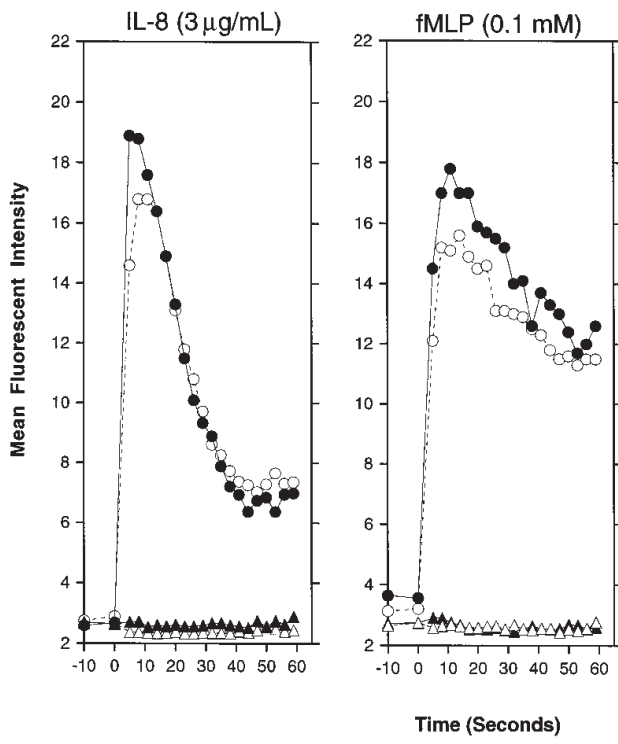

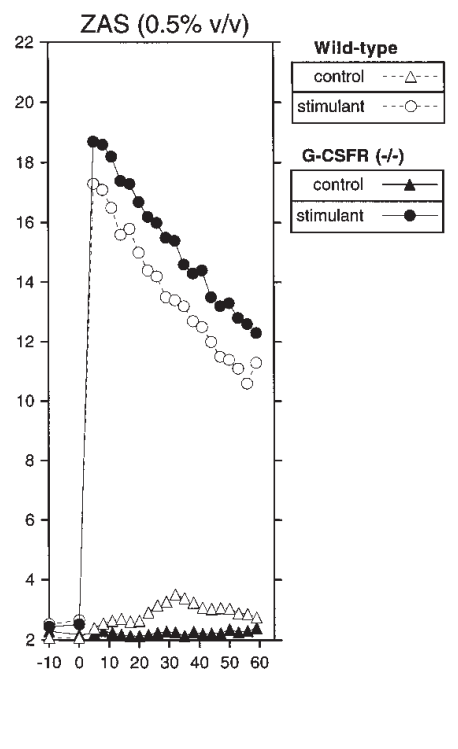




\section{Figure 4}

Superoxide generation. Superoxide dismutase-inhibitable cytochrome c reduction was assessed after stimulation with PMA $(100 \mathrm{ng} / \mathrm{ml})$ by measuring OD at $550 \mathrm{~nm}\left(O D_{550}\right)$. Data represent the mean \pm SD of triplicate determinations. Data are representative of three separate experiments.

marrow PMNs were examined by flow cytometry. The kinetics and magnitude of the increase and subsequent decrease in F-actin content in G-CSFR-deficient PMNs in response to IL-8 were comparable to those for wild-type PMNs (Fig. 7). These data suggest that IL-8-induced actin polymerization in G-CSFR-deficient neutrophils is normal.

$P M N$ recruitment in response to $I L-8$ is impaired in vivo. To determine whether the defect in PMN chemotaxis resulted in impaired PMN emigration in vivo, we examined PMN recruitment into the skin of G-CSFR-deficient mice in response to IL-8. IL-8 (or buffer alone) was injected intradermally. Four hours later, the skin and underlying subcutaneous tissue at the injection site were excised. PMN recruitment was assessed by measuring MPO activity in protein extracts prepared from this tissue and by microscopic examination of $H \& E$ stained tissue; importantly, we have shown previously (22) that the MPO activity of GCSFR-deficient PMNs is normal. As reported previously (41), IL-8 injection into the skin of wild-type mice resulted in nearly an eightfold increase in MPO activity compared with that for mice treated with buffer alone (Fig. 8). In contrast, no significant increase in MPO activity was detected in IL-8-treated G-CSFR-deficient mice. Likewise, histological examination of the skin demonstrated that intradermal injection of IL-8 in the skin provoked a prominent PMN infiltration in wild-type mice but not in GCSFR-deficient mice (data not shown). Although the neutropenia present in G-CSFR-deficient mice might have contributed to the impaired response, the absence of any significant PMN recruitment suggests that IL-8-induced $\mathrm{PMN}$ migration is defective in these mice.

\section{Discussion}

G-CSF is widely used in the treatment of infectious diseases, especially when associated with neutropenia. Although the ability of G-CSF to stimulate PMN produc-

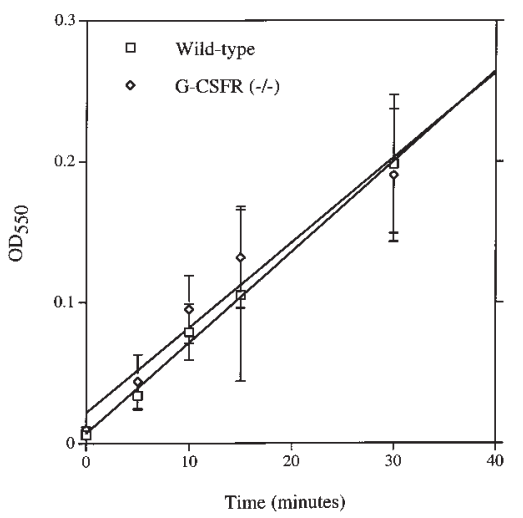

tion is well documented, the biologic importance of GCSF in regulating mature PMN function is less clear. GCSF has been reported to modify a variety of PMN functions, including chemotaxis $(15,16)$, respiratory burst $(8$, $17,18)$, degranulation $(12,13)$, expression of CD11b (6-8) and FcyR1 (9-11), adhesion (6-8), and phagocytosis (14). However, many of these observations were made by comparing the phenotype of PMNs isolated from GCSF-treated compared with untreated healthy volunteers, so that some of the observed effects of G-CSF on PMN function may be due to an indirect mechanism. In this study, we have examined the consequences of the absence of G-CSFR signals on PMN phenotype. We show that GCSFR-deficient PMNs have significant but selective functional defects, indicating that the G-CSF receptor is providing unique signals required for normal PMN function.

Considerable controversy exists as to the importance of cytokine signals in the terminal differentiation of hematopoietic cells. Studies of G-CSF- and GCSFR-deficient mice have demonstrated that the production of morphologically normal mature PMNs is preserved, albeit at reduced levels (21-23). In addition, we recently showed (42) that transduction of primary hematopoietic progenitors with a retrovirus encoding for a chimeric receptor containing the extracellular and transmembrane domains of the G-CSFR fused to the cytoplasmic domain of the erythropoietin receptor was $a$

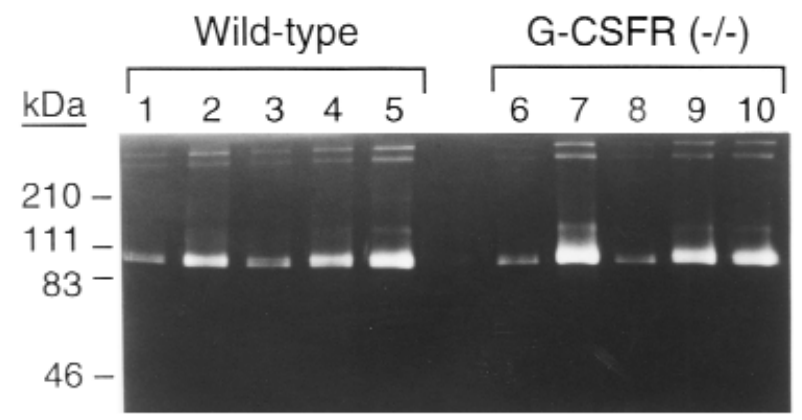

$\boldsymbol{b}$

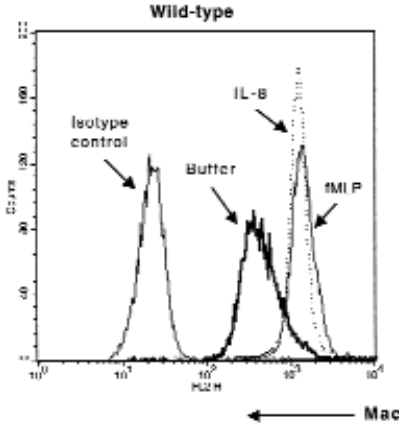

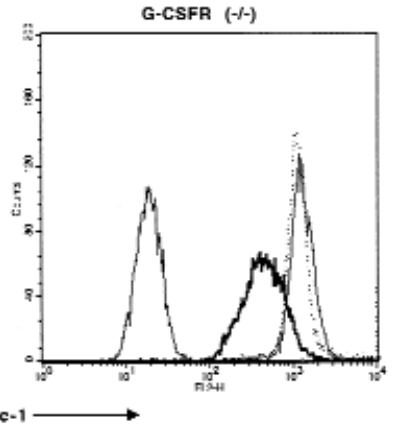

\section{Figure 5}

Degranulation. (a) Gelatinase B release. Bone marrow-purified PMNs were stimulated with buffer alone (lanes 1 and 6 ), fMLP (0.1 mM; lanes 2 and 7), IL-8 $(0.1 \mu \mathrm{g} / \mathrm{ml}$; lanes 3 and 8$)$, IL-8 $(3 \mu \mathrm{g} / \mathrm{ml}$; lanes 4 and 9), or IL-8 $(30 \mu \mathrm{g} / \mathrm{ml}$; lanes 5 and 10$)$, and the amount of gelatinase B released into the supernatant was analyzed by gelatin zymography. Zones of enzymatic activity appear as unstained bands. ( $\boldsymbol{b}$ ) CD11b upregulation. Bone marrow-purified PMNs were stimulated with buffer alone, interleukin-8 (IL-8; $3 \mu \mathrm{g} / \mathrm{ml})$, or $N$-formyl-methionyl-leucyl-phenylalanine ( $f M L P$; $0.1 \mathrm{mM})$, and the expression of CD11b on Gr-1-positive cells was measured by flow cytometry. Data are representative of three separate experiments. 


\section{Figure 6}

Adhesion. An equal number of calcein-AM-loaded bone marrow-purified PMNs were layered onto FBS-coated 96-well plates and stimulated with buffer alone (HBSS), $N$-formyl-methionyl-leucyl-phenylalanine ( $P$ MLP; $0.1 \mathrm{mM})$, ZAS $(0.5 \% \mathrm{vol} / \mathrm{vol}), \mathrm{IL}-8(3 \mu \mathrm{g} / \mathrm{ml})$, or PMA $(100 \mathrm{ng} / \mathrm{ml})$ for $3 \mathrm{~min}$ at $37^{\circ} \mathrm{C}$. Fluorescence (at $538 \mathrm{~nm}$ ) was measured before and after washing to remove nonadherent PMNs. The adhesion index is the fluorescence after washing divided by the fluorescence before washing $(\times 100)$ and represents the percentage of adherent cells. Results were corrected for the percentage of PMNs present in the starting sample. Data represent the mean \pm SD of triplicate determinations and are representative of four separate experiments.

able to support the production of morphologically mature PMNs in response to G-CSF. Collectively, these data indicate that the signals generated by the G-CSFR are not required for terminal granulocytic differentiation. However, the present study clearly demonstrates that the G-CSFR is providing unique signals required for normal PMN function. In similar fashion, GMCSF-deficient mice, while having normal macrophage number and morphology, have a defect in macrophage function that leads to a clinical syndrome with features of pulmonary alveolar proteinosis (43-45).

Priming of the respiratory burst is a well-established effect of G-CSF on PMN function. Pretreatment of PMNs with G-CSF significantly enhances AMLP-induced, but not PMA-induced, respiratory burst (8). Although the molecular mechanism(s) responsible for the priming effect of G-CSF is not known, it has been shown that GCSF does not affect $\mathrm{AMLP}$ receptor number, affinity, or agonist-induced calcium influx (8). In this study, we found that superoxide generation in G-CSFR-deficient PMNs after PMA treatment is comparable to that of wildtype PMNs, indicating that the reduced nicotinamide adenine dinucleotide phosphate (NADPH) oxidase system is intact. However, superoxide generation was not detected consistently after stimulation of wild-type or GCSFR-deficient PMNs with IL-8 and other chemotactic factors, so we were unable to ascertain whether the absence of G-CSFR signals has effects on chemoattractant-induced superoxide generation.

The effects of G-CSF on PMN chemotaxis are controversial. G-CSF is reported to have a modest chemokinetic effect on neutrophils (15), and G-CSF treatment increases PMN migration across vascular endothelium $(15,16)$, but the intraperitoneal injection of G-CSF does not result in a significant accumulation of PMNs (19). Furthermore, PMN migration into the peritoneum in response to thioglycollate or casein injection is nearly normal in G-CSFR-deficient (22) or G-CSF-deficient (21) mice, respectively. In this study, we show that GCSFR-deficient PMNs have a marked defect in their chemotactic response to a broad range of IL-8 concentrations. Significant defects in chemotaxis were also seen in response to $\mathrm{MLLP}, \mathrm{ZAS}$, or MIP-2, demonstrating that this defect is not restricted to IL-8. Initially, we considered the possibility that the chemotaxis defect was due

\section{Figure 7}

IL-8-induced F-actin polymerization. Bone marrow-purified PMNs were stimulated with buffer alone or interleukin-8 $(\mathrm{IL}-8 ; 3 \mu \mathrm{g} / \mathrm{ml})$ for the indicated times, and the F-actin content of Gr-1-positive (granulocytic) cells was measured by flow cytometry. Data are representative of three separate experiments.

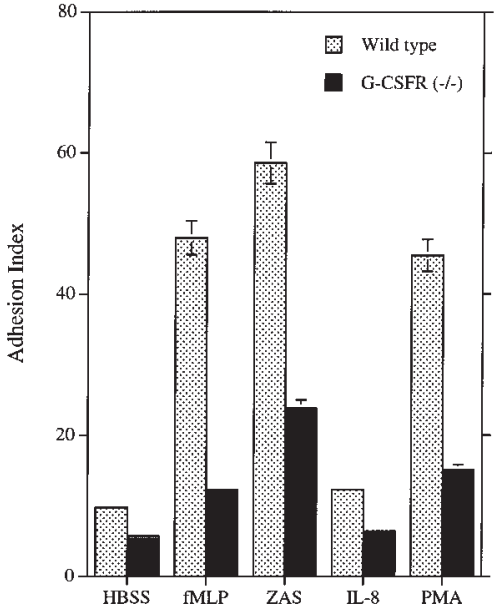

to PMN immaturity in G-CSFR-deficient mice. However, the normal PMN morphology, superoxide generation, and gelatinase $\mathrm{B}$ expression (a marker of terminal PMN differentiation) argue against this possibility. The fact that thioglycollate-elicited G-CSFR-deficient PMNs (which are uniformly mature-appearing) demonstrated similar chemotactic responses provides further evidence that the chemotactic defect in G-CSFR-deficient PMNs is not due to PMN immaturity.

To explore potential mechanisms for the chemotaxis defect, we examined chemoattractant receptor expression and function in G-CSFR-deficient PMNs. G-CSF has been shown to regulate IL-8 receptor expression in PMNs (4). Unlike humans, in mice a single receptor for IL- 8 has been identified, and it is most homologous to the IL-8 type B receptor (CXCR2; refs. 36-38). Cell surface expression of this receptor on G-CSFR-deficient PMNs is normal, indicating that G-CSFR signals are not required for IL-8 receptor expression in mice. Furthermore, IL-8-induced increases in cytoplasmic free $\mathrm{Ca}^{2+}$ and IL-8-induced degranulation, as measured by gelatinase $B$ release and upregulation of cell surface CD11b expression, were normal in G-CSFR-deficient PMNs. Collectively, these data indicate that IL- 8 receptor expression and function are normal.

Reversible actin polymerization is an early required step in neutrophil chemotaxis and adhesion. The molecular

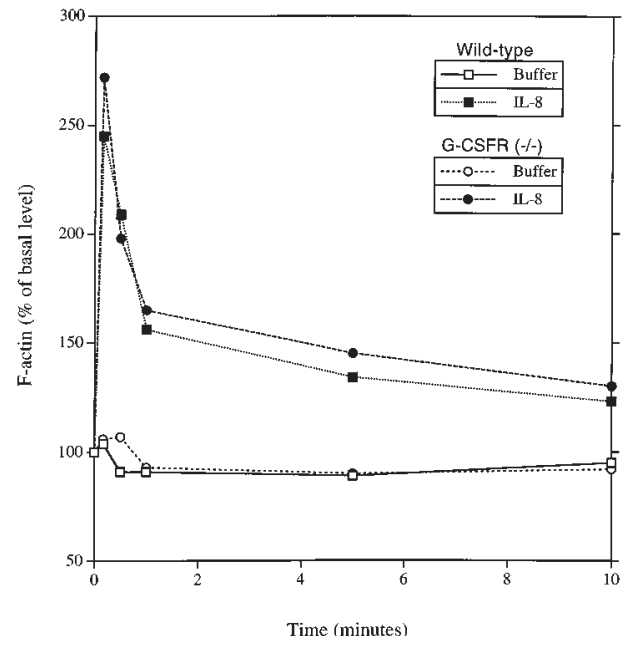




\section{Figure 8}

IL-8-induced PMN emigration into the skin. Interleukin-8 (IL-8; $50 \mathrm{ng}$ ) or buffer alone was injected intradermally into wild-type or G-CSFR-deficient mice (G-CSFR ${ }^{-}-{ }^{-}$. Four hours later, the skin at the injection site was excised and homogenized, and protein extracts were analyzed for MPO activity as described in Methods. MPO content is expressed as the relative MPO activity $\left(\mathrm{OD}_{450}\right)$ per milligram of protein. Six wild-type and nine G-CSFR-deficient mice were analyzed. Data represent the mean $\pm \mathrm{SD}$. ${ }^{*} P<0.05$ compared with buffer alone injected skin. $M P O$, myeloperoxidase.

mechanism by which chemoattractants stimulate actin polymerization in PMNs is incompletely understood. It is clear that neither increases in cytoplasmic free $\mathrm{Ca}^{2+}$ nor activation of protein kinase $\mathrm{C}$ are required for IL-8- or fMLP-induced actin polymerization (46). In this study, we found that IL-8-induced increases and subsequent decreases in F-actin content were normal in G-CSFR-deficient PMNs, demonstrating that G-CSFR signals are not required for actin polymerization and depolymerization.

$\beta 2$-integrin activation on PMNs is thought to play a critical role in PMN adhesion and chemotaxis (47). This conclusion is best exemplified by studies of patients with leukocyte adhesion deficiency (LAD) who have a defect in the gene encoding for the $\beta$ subunit (CD18) of $\beta 2$ integrins (48). LAD is manifest by prominent leukocytosis, failure of PMN emigration to sites of inflammation, and recurrent bacterial infections (49). In vitro, LAD PMNs are deficient in their ability to orient and to migrate in response to chemoattractant gradients (50). In this study, we show impaired chemoattractant-induced adhesion of GCSFR-deficient PMNs to FBS-coated plates. PMN adhesion to FBS-coated plates has been shown to depend largely on the activation of $\beta 2$ integrins (33). Given that the cell surface expression of CD11b (the major $\beta 2$ integrin on neutrophils) on G-CSFR-deficient PMNs is normal and is appropriately upregulated after chemoattractant stimulation, this result suggests that chemoattractant-induced $\beta 2$ integrin activation is impaired.

On resting neutrophils, $\beta 2$ integrins bind poorly to their ligands. In response to various activating stimuli (including chemoattractants), the avidity of $\beta 2$ integrins for their ligands is increased, a process termed "inside-out' signaling (51). The molecular mechanisms responsible for inside-out signaling are incompletely understood. Recently, the Rho family of small-molecular-weight guanosine triphosphatase (GTPase) has been implicated in chemoattractant-induced $\beta 2$-integrin activation (52). Treatment of PMNs with C3 transferase exoenzyme (a specific inhibitor of Rho proteins) blocked chemoattractantinduced chemotaxis and chemoattractant- and PMAinduced adhesion but did not effect chemoattractantinduced calcium flux, degranulation, or superoxide generation $(53,54)$. This pattern of dissociation looks similar to that seen with G-CSFR-deficient PMNs, suggesting that Rho activation may be suppressed in G-CSFR-deficient PMNs. Although G-CSF-induced Rho activation has not been reported, G-CSF stimulation does activate Ras, another small-molecular-weight GTPase (55).

IL-8 is thought to be a major regulator of PMN activation and migration in vivo. Our recent observation that IL-8 administration to G-CSFR-deficient mice failed to induce the expected increase in circulating PMNs suggested a defect in IL-8-induced PMN migration in vivo. Consistent with this conclusion, in the present study we

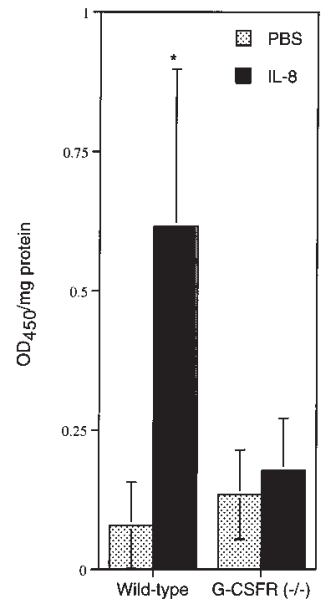

show that PMN recruitment into the skin of GCSFR-deficient mice in response to IL-8 is markedly impaired. G-CSFR-deficient mice are neutropenic; it is therefore possible that the decrease in the level of circulating PMNs may be responsible for the impaired PMN emigration. This possibility seems unlikely for two reasons. First, we have failed to detect any correlation in this assay between the level of circulating PMNs and PMN emigration in wild-type mice (data not shown). Second, despite the neutropenia, thioglycollate injection into the peritoneum of G-CSFR-deficient mice results in the recruitment of a significant number of PMNs $(\sim 30 \%$ that of wild-type mice) (22). Interestingly, this pattern of impaired PMN emigration into the skin but normal emigration into the peritoneum after a inflammatory stimulus is similar to that reported for CD18 ( $\beta 2$ integrin)-deficient mice (56).

In summary, this study demonstrates that G-CSFR signals are required for normal PMN activation. The selective nature of the defects in PMN activation suggests that GCSFR-deficient PMNs may be a valuable model to dissect the signaling pathways used by IL- 8 and other chemoattractants to stimulate specific PMN functions. Of particular interest, the G-CSFR appears to play an important role in the activation of $\beta 2$ integrins by IL- 8 . Because GCSF levels are often elevated in the serum and at inflammatory sites in infected patients $(19,20)$, and given that $\beta 2$ integrins play an important role in PMN activation, these data suggest that G-CSF may significantly modulate the PMN response to IL-8 during infectious challenge.

\section{Acknowledgments}

The authors are grateful to Jennifer Poursine-Laurent and M. Susan Mudd (Washington University) for technical assistance.This work was supported by grants from Monsanto/Searle (to D.C. Link), the Alan A. and Edith L. Wolff Charitable Trust to R.M. Senior, and the National Institutes of Health (HL-47328 to R.M. Senior), and by a Research Fellowship from the Japan Society for the Promotion of Science for Young Scientists (to T. Betsuyaku).

1. Matsushima, K., and Oppenheim,J.J. 1989. Interleukin 8 and MCAF: novel inflammatory cytokines inducible by IL-1 and TNF. Cytokine. 1:2-13.

2. Hoch, R.C., Schraufstätter, I.U., and Cochrane, C.G. 1996. In vivo, in vitro, and molecular aspects of interleukin-8 and the interleukin-8 receptors. J. Lab. Clin. Med. 128:134-145.

3. Matsukawa, A., et al. 1995. Neutrophil accumulation and activation by homologous Il-8 in rabbits. J. Immunol. 154:5418-5425. 
4. Lloyd, A.R., et al. 1995. Granulocyte-colony stimulating factor and lipopolysaccharide regulate the expression of interleukin 8 receptors on polymorphonuclear leukocytes. J. Biol. Chem. 270:28188-28192.

5. Anderlini, P., Przepiorka, D., Champlin, R., and Korbling, M. 1996. Biologic and clinical effects of granulocyte colony-stimulating factor in normal individuals. Blood. 88:2819-2825.

6. Hakansson, L., et al. 1997. Effects of in vivo administration of G-CSF on neutrophil and eosinophil adhesion. Br. J. Haematol. 98:603-611.

7. Yong, K.L., and Linch, D.C. 1992. Differential effects of granulocyte- and granulocyte-macrophage colony-stimulating factors (G- and GM-CSF) on neutrophil adhesion in vitro and in vivo. Eur. J. Haematol. 49:251-259.

8. Yuo, A., et al. 1989. Recombinant human granulocyte colony-stimulating factor as an activator of human granulocytes: potentiation of responses triggered by receptor-mediated agonists and stimulation of C3bi receptor expression and adherence. Blood. 74:2144-2149.

9. Kerst, J.M., et al. 1993. Recombinant granulocyte colony-stimulating factor administration to healthy volunteers: induction of immunophenotypically and functionally altered neutrophils via an effect on myeloid progenitor cells. Blood. 82:3265-3272.

10. Kerst, J.M., et al. 1993. Granulocyte colony-stimulating factor induces hFc gamma RI (CD64 antigen)-positive neutrophils via an effect on myeloid precursor cells. Blood. 81:1457-1464.

11. Gericke, G.H., et al. 1995. Mature polymorphonuclear leukocytes express high-affinity receptors for IgG (Fc gamma RI) after stimulation with granulocyte colony-stimulating factor (G-CSF). J. Leukocyte Biol. 57:455-461.

12. Xu, S., Hoglund, M., and Venge, P. 1996. The effect of granulocyte colonystimulating factor (G-CSF) on the degranulation of secondary granule proteins from human neutrophils in vivo may be indirect. Br. J. Haematol. 93:558-568.

13. de Haas, M., et al. 1994. Granulocyte colony-stimulating factor administration to healthy volunteers: analysis of the immediate activating effects on circulating neutrophils. Blood. 84:3885-3894.

14. Hoglund, M., Hakansson, L., and Venge, P. 1997. Effects of in vivo administration of G-CSF on neutrophil functions in healthy volunteers. Eur. J. Haematol. 58:195-202.

15. Smith, W.B., Gamble, J.R., and Vadas, M.A. 1994. The role of granulocytemacrophage and granulocyte colony-stimulating factors in neutrophil transendothelial migration: comparison with interleukin-8. Exp. Hematol. 22:329-334

16. Yong, K.L. 1996. Granulocyte colony-stimulating factor (G-CSF) increases neutrophil migration across vascular endothelium independent of an effect on adhesion: comparison with granulocyte-macrophage colony-stimulating factor (GM-CSF). Br. J. Haematol. 94:40-47.

17. Yuo, A., Kitagawa, S., Ohsaka, A., Saito, M., and Takaku, F. 1990. Stimulation and priming of human neutrophils by granulocyte colony-stimulating factor and granulocyte-macrophage colony-stimulating factor: qualitative and quantitative differences. Biochem. Biophys. Res. Commun. 171:491-497.

18. Khwaja, A., Carver, J.E., and Linch, D.C. 1992. Interactions of granulocytemacrophage colony-stimulating factor (CSF), granulocyte CSF, and tumo necrosis factor alpha in the priming of the neutrophil respiratory burst. Blood. 79:745-753.

19. Metcalf, D., Robb, L., Dunn, A.R., Mifsud, S., and Di Rago, L. 1996. Role of granulocyte-macrophage colony-stimulating factor and granulocyte colonystimulating factor in the development of an acute neutrophil inflammatory response in mice. Blood. 88:3755-3764.

20. Cebon, J., Layton, J.E., Maher, D., and Morstyn, G. 1994. Endogenous haemopoietic growth factors in neutropenia and infection. Br. J. Haematol. 86:265-274.

21. Lieschke, G.J., et al. 1994. Mice lacking granulocyte colony-stimulating factor have chronic neutropenia, granulocyte and macrophage progenitor cell deficiency, and impaired neutrophil mobilization. Blood. 84:1737-1746.

22. Liu, F., Wu, H.Y., Wesselschmidt, R., Kornaga, T., and Link, D. 1996. Impaired production and increased apoptosis of neutrophils in granulocyte colony-stimulating factor receptor deficient mice. Immunity. 5:491-501.

23. Liu, F., Poursine-Laurent, J., Wu, H., and Link, D. 1997. IL-6 and the G-CSF receptor are major independent regulators of granulopoiesis in vivo but are not required for lineage commitment or terminal differentiation. Blood. 90:2583-2590

24. Liu, F., Poursine-Laurent, J., and Link, D.C. 1997. The granulocyte colonystimulating factor receptor is required for the mobilization of murine hematopoietic progenitors into peripheral blood by cyclophosphamide or interleukin-8 but not Flt-3 ligand. Blood. 90:2522-2528

25. Sugawara, T., Miyamoto, M., Takayama, S., and Kato, M. 1995. Separation of neutrophils from blood in human and laboratory animals and comparison of the chemotaxis. J. Pharmacol. Toxicol. Methods. 33:91-100.

26. Wang, J., et al. 1996. Detection of mouse IL-8 receptor homologue expression on peripheral blood leukocytes and mature myeloid lineage cells in bone marrow. J. Leukoc. Biol. 60:372-381.

27. Rosen, H., and Gordon, S. 1987. Monoclonal antibody to the murine type 3 complement receptor inhibits adhesion of myelomonocytic cells in vitro and inflammatory cell recruitment in vivo. J. Exp. Med. 166:1685-701.
28. Lindberg, F.P., et al. 1994. Rh-related antigen CD47 is the signal-transducer integrin-associated protein. J. Biol. Chem. 269:1567-1570.

29. Lowell, C.A., Fumagalli, L., and Berton, G. 1996. Deficiency of Src family kinases $\mathrm{p} 59 / 61^{\text {hck }}$ and $\mathrm{p} 58^{\mathrm{c} \text {-fgr }}$ results in defective adhesion-dependent neutrophil functions. J. Cell Biol. 133:895-910.

30. Rot, A. 1991. Chemotactic potency of recombinant human neutrophil attarctant/activation protein-1 (interleukin-8) for polymorphonuclear leukocytes of different species. Cytokine. 3:21-27.

31. Brenner, C.A., Adler, R.R., Rappollee, D.A., Pederson, R.A., and Werb, Z. 1989. Genes for extracellular matrix-degrading metalloproteinases and their inhibitor, TIMP, are expressed during early mammalian development. Genes Dev. 3:848-859.

32. Mayo, L.A., and Curnutte,J.T. 1990. Kinetic microplate assay for superoxide production by neutrophils and other phagocytic cells. Methods Enzymol. 186:567-575.

33. Jones, S.L., Knaus, U.G., Bokoch, G.M., and Brown, E. 1998. Two signaling mechanism for activation of $\alpha_{M} \beta_{2}$ activity in polymorphonuclear neutrophils. J. Biol. Chem. 273:10556-10566.

34. Kim, C.H., and Broxmeyer, H.E. 1998. In vitro behavior of hematopoietic progenitor cells under the influence of chemoattractants: stromal cell-derived factor-1, steel factor, and the bone marrow environment. Blood. 91:100-110.

35. Liu, Z., et al. 1998. Gelatinase B-deficient mice are resistant to experimental bullous pemphigoid. J. Exp. Med. 188:475-482.

36. Cacalano, G., et al. 1994. Neutrophil and B cell expansion in mice that lack the murine IL-8 receptor homolog [published erratum appears in Science, 1995, 270:365]. Science. 265:682-684.

37. Bozic, C.R., et al. 1994. The murine interleukin 8 type B receptor homologue and its ligands. Expression and biological characterization. J. Biol. Chem. 269:29355-29358

38. Lee, J., et al. 1995. Chemokine binding and activities mediated by the mouse IL-8 receptor. J. Immunol. 155:2158-2164.

39. Borregaard, N., and Cowland, J.B. 1997. Granules of the human neutrophilic polymorphonuclear leukocyte. Blood. 89:3503-3521.

40. Jones, D.H., et al. 1990. Subcellular distribution and mobilization of MAC1 (CD11b/CD18) in neonatal neutrophils. Blood. 75:488-498.

41. Larsen, C.G., Anderson, A.O., Oppenheim, J.J., and Matsushima, K. 1989. Production of interleukin- 8 by human dermal fibroblasts and keratinocytes in response to interleukin-1 to tumor necrosis factor. Immunology. 68:31-36.

42. Jacob, J., Haug, J.S., Raptis, S., and Link, D.C. 1998. Specific signals generated by the cytoplasmic domain of the G-CSF receptor are not required for GCSF-dependent granulocytic differentiation. Blood. 92:353-361.

43. Huffman, J.A., Hull, W.M., Dranoff, G., Mulligan, R.C., and Whitsett, J.A. 1996. Pulmonary epithelial cell expression of GM-CSF corrects the alveolar proteinosis in GM-CSF-deficient mice. J. Clin. Invest. 97:649-655.

44. Dranoff, G., et al. 1994. Involvement of granulocyte-macrophage colonystimulating factor in pulmonary homeostasis. Science. 264:713-716.

45. Nishinakamura, R., et al. 1996. The pulmonary alveolar proteinosis in granulocyte macrophage colony-stimulating factor/interleukins $3 / 5 \mathrm{Bc}$ receptor-deficient mice is reversed by bone marrow transplantation. J. Exp. Med. 183:2657-2662.

46. Sham, R.L., Phatak, P.D., Ihne, T.P., Abboud, C.N., and Packman, C.H. 1993. Signal pathway regulation of interleukin-8-induced actin polymerization in neutrophils. Blood. 82:2546-2551.

47. Brown, E.J. 1997. Adhesive interactions in the immune system. Trends Cell Biol. 7:289-295.

48. Dana, N., Todd, R.F.,3d., Pitt, J., Springer, T.A., and Arnaout, M.A. 1984. Deficiency of a surface membrane glycoprotein (Mo1) in man.J. Clin. Invest. 73:153-159.

49. Anderson, D.C., et al. 1985. The severe and moderate phenotypes of heritable Mac-1, LFA-1 deficiency: their quantitative definition and relation to leukocyte dysfunction and clinical features. J. Infect. Dis. 152:668-689.

50. Anderson, D.C., and Springer, T.A. 1987. Leukocyte adhesion deficiency: an inherited defect in the Mac-1, LFA-1, and p150, 95 glycoproteins. Am. Rev. Med. 38:175-194.

51. Kolanus, W., and Seed, B. 1997. Integrins and inside-out signal transduction: converging signals from PKC and PIP3. Curr. Opin. Cell Biol. 9:725-731.

52. Faull, R.J., and Ginsberg, M.H. 1996. Inside-out signaling through integrins. J. Am. Soc. Nephrol. 7:1091-1097.

53. Stasia, M.J., Jouan, A., Bourmeyster, N., Boquet, P., and Vignais, P.V. 1991. ADP-ribosylation of a small size GTP-binding protein in bovine neutrophils by the $\mathrm{C} 3$ exoenzyme of Clostridium botulinum and effect on the cell motility. Biochem. Biophys. Res. Commun. 180:615-622.

54. Laudanna, C., Campbell, J.J., and Butcher, E.C. 1996. Role of Rho in chemoattractant-activated leukocyte adhesion through integrins. Science. 271:981-983.

55. Bashey, A., Healy, L., and Marshall, C.J. 1994. Proliferative but not nonproliferative responses to granulocyte colony-stimulating factor are associated with rapid activation of the $\mathrm{p} 21 \mathrm{ras} / \mathrm{MAP}$ kinase signalling pathway. Blood. 83:949-957.

56. Mizgerd, J.P., et al. 1997. Neutrophil emigration in the skin, lungs and peritoneum: different requirement for CD11/CD18 revealed by CD18-deficient mice. J. Exp. Med. 186:1357-1364. 